archives-ouvertes

\title{
Progress in OCT-based Through Silicon Via (TSV) metrology
}

\author{
Alain Bosseboeuf
}

\section{To cite this version:}

Alain Bosseboeuf. Progress in OCT-based Through Silicon Via (TSV) metrology. Frontiers in Optics, 2019, Washington, France. pp.JW4A.115, 10.1364/FIO.2019.JW4A.115 . hal-02343398

\section{HAL Id: hal-02343398 \\ https: / hal.archives-ouvertes.fr/hal-02343398}

Submitted on 2 Nov 2019

HAL is a multi-disciplinary open access archive for the deposit and dissemination of scientific research documents, whether they are published or not. The documents may come from teaching and research institutions in France or abroad, or from public or private research centers.
L'archive ouverte pluridisciplinaire HAL, est destinée au dépôt et à la diffusion de documents scientifiques de niveau recherche, publiés ou non, émanant des établissements d'enseignement et de recherche français ou étrangers, des laboratoires publics ou privés. 


\title{
Progress in OCT-based Through Silicon Via (TSV) metrology
}

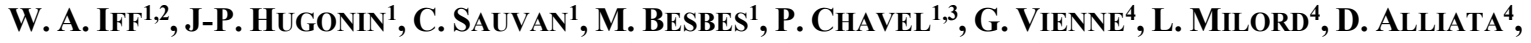 \\ E. HERTH ${ }^{2}$, P. COSTE ${ }^{2}$, A. BOSSEBOEUF ${ }^{2}$ \\ ${ }^{1}$ Laboratoire Charles Fabry, Institut d'Optique Graduate School, CNRS, Université Paris-Saclay, 91127 Palaiseau cedex, France \\ ${ }^{2}$ Centre de Nanosciences et de Nanotechnologies, CNRS, Univ. Paris-Sud, Université Paris Saclay, C2N-10 Bd Thomas Gobert F-91120 \\ Palaiseau, France \\ ${ }^{3}$ Laboratoire Hubert Curien (Université Jean Monnet de Saint-Etienne - Université de Lyon, CNRS, IOGS), 18 rue Professeur Benoît Lauras, \\ 42000 Saint-Etienne, France \\ ${ }^{4}$ UnitySC, 611 rue Aristide Berges, ZA Pre Millet, Montbonnot Saint Martin F-38330, France \\ *pierre.chavel@institutoptique.fr
}

\begin{abstract}
We report on progress in Time-Domain OCT (optical coherence tomography) applied to TSV (vertical interconnect accesses in silicon, enabling stacking of devices). Transitioning from the common scalar approach to an electromagnetic one, and combining it with a damped least squares approach, we enhance the accuracy of TSV height measurements. (c) 2019 The Author(s) OCIS codes: $120.0120,100.3190,110.4500,120.2830,120.4825,130.5990$
\end{abstract}

\section{Time Domain OCT applied to TSVs}

Time-Domain OCT (fig. 1) is a widely-used method for measuring 3D structures. One important application nowadays is the metrology of empty TSVs (interconnects etched into semiconductors, enabling 3D integration and thus more compact electronic chips) [1-3]. The main TSV parameter to be monitored during the fabrication process is its height in order to ensure a full opening of the TSVs as well as a uniform via protrusion over the wafer in later fabrication steps.

Schematic draft of the time-domain OCT apparatus for TSV measurements

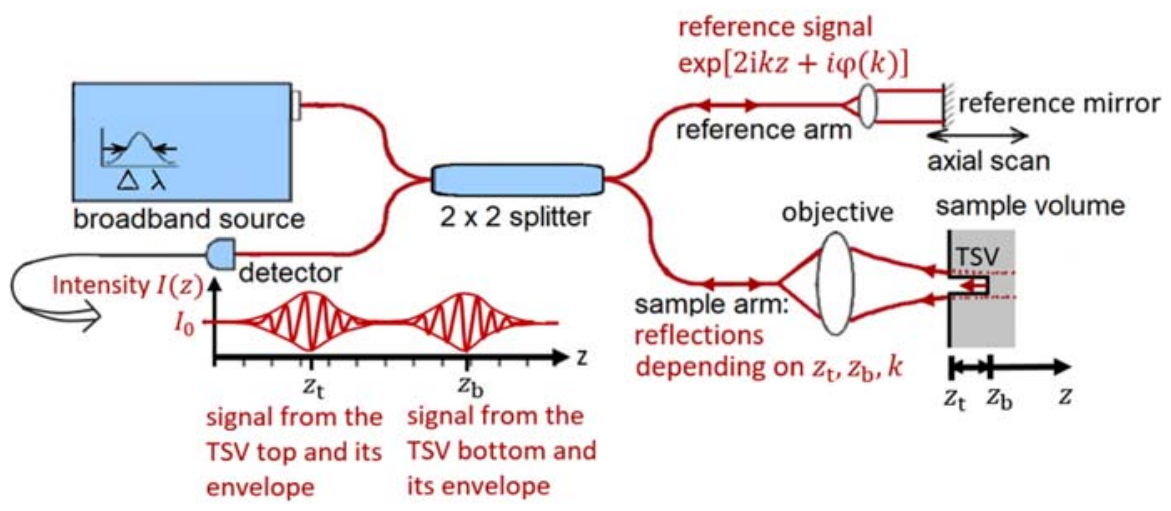

Figure 1. Scheme of a time domain OCT device for TSV measurements [2-4]. The motion of the reference mirror enables longitudinal resolution. The detector measures the intensity $I(z)$ versus the z-position of the reference mirror; $I_{0}$ is the z-independent background intensity. The sample is illuminated by a linearly polarized Gaussian beam which covers the TSV plus the surrounding substrate so that the signals from the TSV top and bottom have a similar magnitude. The reference signal is $\exp [2 \mathrm{i} k z+\mathrm{i} \varphi(k)]$, considering dispersion-induced phase shifts $\varphi(k) ; k$ is the modulus of the vacuum wave vector.

\section{Modeling of the optical system and interferogram}

A common approach for modeling TSVs is ray tracing, assuming propagation in air with a refractive index of $\mathrm{n}=1$. In contrast to this, our novel approach relies on electromagnetic modeling [3]. It starts with the recording of a reference interferogram at a planar substrate and its subsequent Fourier transform, yielding the spectrum applied at the TSV; dispersion that is intrinsic to the interferometer is thus considered. The frequency components are then separately propagated in the TSV by a modal approach. In the case of TSVs of sufficient aspect ratio, only the fundamental mode with its propagation constant needs to be considered between TSV top and bottom, allowing fast calculations. The interferogram is then simulated by the addition of the reflection from the reference mirror and the numeric integration over the spectrum. 


\section{Evaluation of the OCT interferograms}

Commonly, the OCT signal recorded at the TSV (fig. 1) is evaluated by the detection of the envelopes of the intensity modulations and their maximum; the z-distance between these maxima is then associated with the depth of the object - here the TSV [4].

In contrast to this, our novel approach is based on the solution of an inverse problem, matching measured and simulated OCT interferograms. For this, we define a least squares-based merit function, which depends on the TSV parameters, and minimize it by the Marquardt-Levenberg algorithm. The TSV parameters yielding the minimum of the merit function are the desired values.

In view of an efficient computational procedure, our merit function is formulated in Fourier space, where the propagation at a TSV is performed by the multiplication of the complex Fourier coefficients with exponential factors. For this, we Fourier transform the measured OCT signals. Since the domain in direct space is physically restricted to a finite-size region where the OCT signals are meaningful, we handle a set of discrete Fourier coefficients. To enhance the accuracy and reduce the influence of noise, we apply a bandpass filter to the recorded spectrum as well as the measured Fourier transformed interferogram, adding the source spectrum support as a constraint. This allows also a further acceleration of our computations, enabling "real-time data" evaluations $(2-3 \mathrm{~s}$ with our current hardware).

Fig. 2 presents a measured OCT interferogram after application of the bandpass filter (violet) as well as the simulated interferogram as a match (green). They are depicted here in direct space for illustration purposes; the algorithm itself does not require such measure and operates in reciprocal space. Apart from minor deviations, attributable to small remaining noise, the measured and simulated interferograms match well.

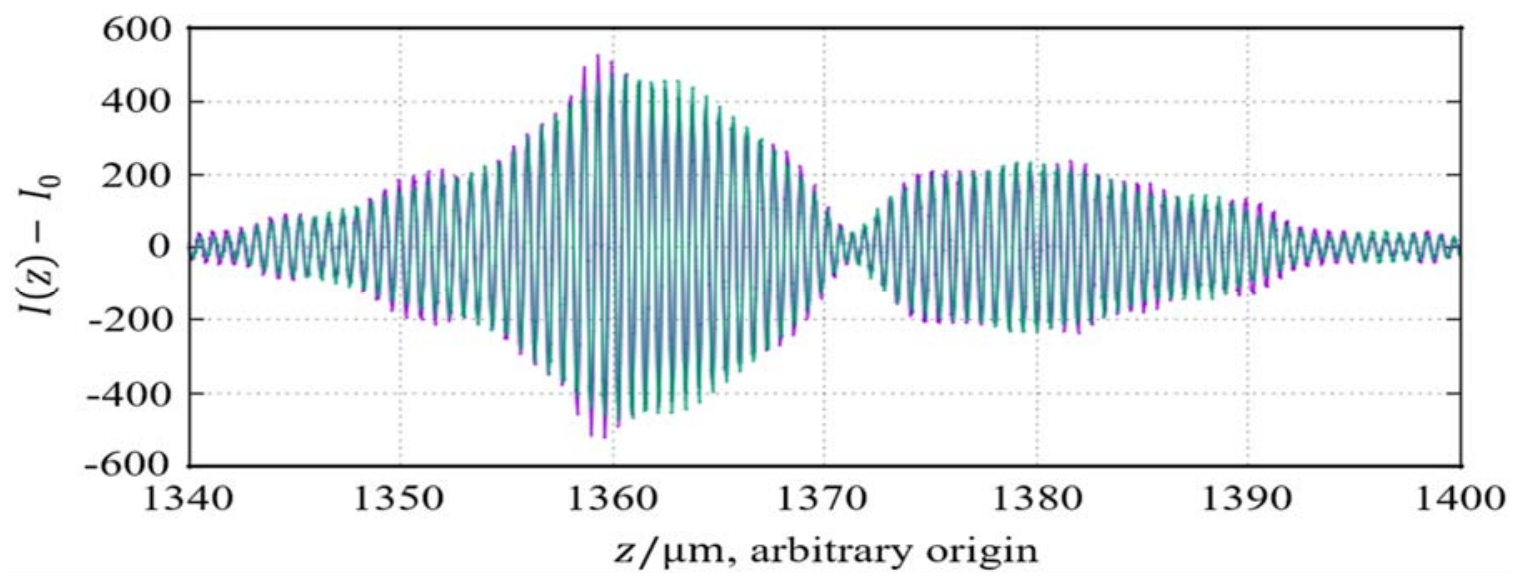

Figure 2. Example of an OCT interferogram in the case of linear polarization at a circular TSV of diameter $D=12 \mu \mathrm{m}$ and height $H=20 \mu \mathrm{m}$. Violet color in the background: measured signal after bandpass filtering. Green: interferogram simulated with best matching z-positions of TSV top and bottom. The interferogram is presented here in direct space for illustration purposes; the computations have been performed in reciprocal space. The fringe pattern on the left belongs to the TSV top and the one on the right to the bottom. The patterns overlap and interfere destructively in the region of overlap in this example; local intensity maxima of the signal on the left coincide with intensity minima of the signal on the right and vice versa, reducing the contrast in the region of overlap in this example.

Comparisons of our estimated TSV heights with those from the conventional method reveal a progress in reliability, accuracy, and reproducibility. This can be used for a more realistic, detailed modeling, also automatically including the interference of the fringe patterns from TSV top and bottom, which can overlap and interact.

\section{References}

[1] International Technology Roadmap for Semiconductors (ITRS), Assembly and Packaging, (Semiconductor Industry Association, Washington DC, USA, 2009).

[2] N. Devanciard et al, "Through Silicon Via Process Characterization by Integrated Inspection/Metrology Solutions in Visible and Infrared Domain", in $26^{\text {th }}$ Annual SEMI Advanced Semiconductor Manufacturing Conference (ASMC), (IEEE, New York, USA, 2015$)$, pp. 90-95.

[3] W. Iff et al, "Simulation of a Time-Domain OCT device for Through Silicon Via (TSV) fabrication", in TOM 6 in EOS annual meeting 2018, (European Optical Society, Joensuu, Finland, 2018), pp. 290-291.

[4] R. Wilhelm, A. Courteville, F. Garcia, "A novel, low coherence fibre optic interferometer for position and thickness measurements with unattained accuracy”, in Proc. SPIE 6189, Optical sensing II (SPIE, Bellingham, USA, 2006), item 618918. 